\title{
A STUDY OF NON-LINEAR VIBRATIONAL BEHAVIOR OF CRACKED STRUCTURES BY THE FINITE ELEMENT METHOD
}

\author{
Tzuo-Liang Luo \\ Ph.D. Candidate, Institute of Mechanical Engineering, National ChungHsing University 250, Kuo-Kuang Rd.,Taichung, \\ Taiwan 402, R.O.C \\ James Shih-Shyn Wu \\ Ph.D., Professor, Institute of Mechanical Engineering, National ChungHsing University 250, Kuo-Kuang Rd., Taichung, \\ Taiwan 402, R.O.C \\ Jui-Pin Hung \\ Ph.D., Associate Professor, Department of Mechanical Engineering, Nanya Institute of Technology 414, Sec. 3, Jung- \\ Shan E. Rd., Chungli, Taiwan 320, R.O.C.
}

Follow this and additional works at: https://jmstt.ntou.edu.tw/journal

Part of the Mechanical Engineering Commons

\section{Recommended Citation}

Luo, Tzuo-Liang; Wu, James Shih-Shyn; and Hung, Jui-Pin (2005) "A STUDY OF NON-LINEAR VIBRATIONAL BEHAVIOR OF CRACKED STRUCTURES BY THE FINITE ELEMENT METHOD," Journal of Marine Science and Technology. Vol. 13: Iss. 3, Article 3.

DOI: $10.51400 / 2709-6998.2124$

Available at: https://jmstt.ntou.edu.tw/journal/vol13/iss3/3

This Research Article is brought to you for free and open access by Journal of Marine Science and Technology. It has been accepted for inclusion in Journal of Marine Science and Technology by an authorized editor of Journal of Marine Science and Technology. 


\title{
A STUDY OF NON-LINEAR VIBRATIONAL BEHAVIOR OF CRACKED STRUCTURES BY THE FINITE ELEMENT METHOD
}

\author{
Tzuo-Liang Luo*, James Shih-Shyn Wu**, and Jui-Pin Hung***
}

Key words: breathing crack, crack detection, bilinear vibrational theory.

\section{ABSTRACT}

Numerical and experimental investigations provide a link to the location and size of cracks caused by natural vibrations. Cracks may generally result in the variation of structural stiffness and hence enable structures to vibrate nonlinearly. In order to understand the vibrational behavior of crack structures accurately, the study proposes a general and efficient algorithm based on the finite element assumptions and the bilinear vibrational theory. All formulae are derived from the time domain properly and may apply to the overall non-linear motion cycle completely. The contact effect is also considered by introducing the degree of penetration on the cracked surface. By assessing the variation of natural frequencies in crack open and closed modes, changes in the dynamic characteristics of cracked structures are investigated. A single beam and a spatial rotor blade structure are used to demonstrate the validity of the current method. Results in estimation with the variation of vibrational behaviors are significant compared with those available from experiments as well as some other numerical algorithms. Conseuqently, it is obviously found that the current algorithm allows the prediction of the location and the magnitude of cracks more efficient and significant than before. Further extension of the current method to other related fields is highly suggested.

\section{INTRODUCTION}

Small cracks or defects produced in mechanical components during the manufacturing process will form discontinuities in their geometry, which will also result in a variation of stiffness and hence affect the dynamic characteristics in such a way as to make them difficult to predict. For reasons of safety or other applications,

Paper Submitted 12/30/04, Accepted 03/17/05. Author for Correspondence: James Shih-ShynWu.E-mail: sswu@dragon.nchu.edu.tw.

*Ph.D. Candidate, Institute of Mechanical Engineering, National ChungHsing University 250, Kuo-Kuang Rd.,Taichung, Taiwan 402, R.O.C.

**Ph.D., Professor, Institute of Mechanical Engineering, National ChungHsing University 250, Kuo-Kuang Rd., Taichung, Taiwan 402, R.O.C.

***Ph.D., Associate Professor, Department of Mechanical Engineering, Nanya Institute of Technology 414, Sec. 3, Jung-Shan E. Rd., Chungli, Taiwan 320, R.O.C. it is therefore necessary to predict the dynamic characteristics of a mechanical component with embedded microcracks. An examination of the changes in dynamic characteristics can thus be the justification for the inspection of a cracked structure. For this purpose, different approaches based on analytical or numerical analysis have been adopted to investigate the dynamic characteristics of cracked beam structures. In some of these studies, both sides of the crack were assumed to be in a constantly open state $[10,17]$. However, whether the crack front remains in an open or closed state during vibration is influenced by the amplitude limitation or mutual collision of the crack front surface. When the crack tip closes and opens alternately in line with the vibration, it is referred to as a "breathing crack". A cracked structure with such a breathing crack may thus exhibit non-linear characteristics to different degrees $[5,18]$, depending on the vibration amplitude and mode shape. This increases the complexity of the situation when investigating vibrations in damaged structures, especially the modeling of a breathing crack. Many researchers $[1,6,13,15,16]$ have introduced an elastic rotational spring to represent the discontinuous stiffness or local flexibility at the cracked section. The spring constant is usually derived from fracture mechanics [1, 13]. Another approach using finite element analysis has been verified to be efficient in dealing with the dynamic behavior of a complicate cracked structure. For this kind of numerical analysis [8, 11, 12], the construction of the stiffness matrix of a finite element beam model, in particular, a cross section with cracks, is a necessity. To consider the variation in local stiffness due to the presence of the crack, Gounaris [8] and Khiem [11], respectively, proposed a crack compliance matrix to conjugate with other intact beam sections in the FE model. Based on this concept, Khiem [12] further developed a dynamic stiffness matrix method to model a multiple cracked beam. As in the analytical approach, the cracks were also modeled by a rotational spring element [1] but with different degrees of stiffness depending on structural attributes such as elastic 
properties and the crack depth. However as revealed in certain references $[4,9]$, a breathing crack experiencing vibration will exhibit different kinds of structural stiffness before and after the crack surface comes into contact, and hence cause a change in vibration behavior, appearing as a bilinear vibration characteristic. The modeling of the crack using a single equivalent elastic spring element thus cannot sufficiently describe the bilinear vibration characteristic and the effect of contact on the dynamic behavior. Generally, such a contact effect comes from the mutual contact of the crack in the closing period including the increase in contact stiffness and the constraints on the motion of the cracked section. To ensure accurate prediction of the dynamic behavior, this additional contact effect should be taken into consideration in computation.

Economy and safety are the two main factors, which should be considered during the design of a turbine blade. A blade-shaped component is required not only to be very light, but also to be sufficiently strong. Cracks caused by vibrations are responsible for most blade failures. Reducing the vibration of the blade and lowering its stress is a rigorous challenge. The dynamic analysis of repetitious engineering structures is greatly simplified by assuming a perfect periodicity. Unfortunately, this mathematical idealization is invalid due to unavoidable manufacturing and material defects. On account of Manufacturing flaws or cyclic fatigue during operation, cracks frequently appear in rotating machinery [2,7]. A blade crack may cause a local change in the flexibility. As noted by several investigators [20], a local defect may be introduced into the nonlinear system. Most types of crack in rotating machinery are surface cracks, but it is difficult to emphasize fracture mechanics in this model. To make the analysis easier, the investigation of a two-dimensional problem using a model of a crack extending over the entire chord is necessary. Some researchers [14, 20] have, using a two-dimensional crack model, studied the vibrational behavior of a blade, beam and rotor with a crack. In this study, a new simplified method combining the finite element method (FEM) and bilinear vibration theory is proposed in order to investigate the dynamic characteristics of a cracked structure. Analysis is carried out in the time domain to consider the effect of the motion constraint on the behavior of the vibration. The proposed method is validated by the comparison of current and previously published results. Through the use of this numerical analysis, the effects of the location and depth of the crack on the changes in the vibrational frequencies and mode shape of a cracked structure are investigated. The results predicted by this method can further be applied as a method of crack identification of cracked structures.

\section{THEORY AND FORMULATION OF CONSTRAINED MOTION}

In practice, the vibrational behavior of a crack structure will be affected owing to the displacement constraints on the crack sides. Any finite element nodes on both sides of the crack surface must be controlled when the crack sides close or open during vibration. Therefore, the bilinear vibration behavior of a cracked structure must be simulated as a three-dimensional system with proper motion constraints. In the following, the bilinear natural frequency of a vibrating system with proper displacement constraints is derived.

\section{Period of bilinear motion}

Consider a multi-degree-of-freedom system, the motion equations can be expressed in terms of the matrix form

$[M]\{X\}+[K]\{X\}=0$

A well-known solution to the Eq. (1) at $i^{\text {th }}$ mode is

$x_{j}=x_{o j} \sin \omega t$,

Rewrite Eq. (1) into the following form

$m_{i j} \frac{d \dot{x}_{j}}{d x_{j}}+\frac{k_{i j} x_{j}}{\dot{x}_{j}}=0$

By integrating Eq. (3), we have

$$
\begin{aligned}
\int_{0}^{\dot{x}} m_{i j} \dot{x}_{j} d \dot{x}_{j} & =\int_{-x_{0 j}}^{x_{j}}-k_{i j} x_{j} d x_{j}=\frac{1}{2} m_{i j} \dot{x}_{j}^{2} \\
& =\frac{1}{2} k_{i j}\left(x_{0 j}^{2}-x_{j}^{2}\right)
\end{aligned}
$$

The above Eq. (4) can be written in matrix form as

$$
\frac{1}{2}[m]\left\{\dot{x}^{2}\right\}=\frac{1}{2}[k]\left(\left\{x_{0}^{2}\right\}-\left\{x^{2}\right\}\right)
$$
yields

Letting $[\hat{k}]=[m]^{-1}[k]$ and substituting into Eq. (5),

$$
\left\{\dot{x}^{2}\right\}=[\hat{k}]\left(\left\{x_{0}^{2}\right\}-\left\{x^{2}\right\}\right)
$$

The velocity term in above equation can be further expressed as

$$
\begin{aligned}
& \dot{x}_{j}=\left[\hat{k}_{i j}\left(x_{0 j}^{2}-x_{j}^{2}\right)\right]^{\frac{1}{2}}=\left[\hat{k}_{i j}\right]^{\frac{1}{2}} \sqrt{\left(x_{0 j}^{2}-x_{j}^{2}\right)} \\
& =\kappa_{i j} \sqrt{\left(x_{0 j}^{2}-x_{j}^{2}\right)}
\end{aligned}
$$


where $[\kappa]=\left[[m]^{-1}[k]\right]^{\frac{1}{2}}$

Shown in Figure 1 is the mass $i$ vibrating freely with amplitude $x_{0}$ in certain direction but with an elastic boundary placed at $x_{c i}$ in opposite direction. When the displacement is constrained at $x_{c i}$, the mass may penetrate into the boundary with a depth of $x_{p}$ after contact with the elastic boundary. At this point, a bilinear motion will occur under this condition and the relationship of $x$ and $\dot{x}$ will display an unsymetrical motion depicted in the phase plane of Figure 2, which is quite different from a full ellipse type of free vibration. The period to complete one cyclic motion consists of two parts: free motion period and constrained motion period. This can be estimated from the following integrating form.

$$
T_{i}=2 \oint_{\Gamma_{i}} d t=2\left(T_{-i}+T_{+i}\right)
$$

and $T_{-i}=\oint_{\Gamma_{i-}} d t=\int_{x_{c i}}^{-x} 0 i \frac{1}{x_{-}} d x_{-}$,

$T_{+i}=\oint_{\Gamma_{i+}} d t=\int_{x_{p}}^{x^{c i}} \frac{1}{x_{+}} d x_{+}$

In above equations, $\Gamma_{i}$ represent the motion trajec-

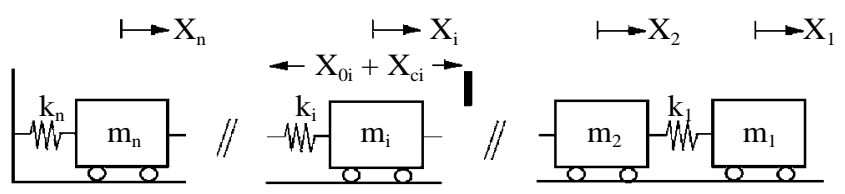

Fig. 1. Schematic showing of a multi-degree-of-freedom system. A constrained boundary is located at $x_{c i}$ to restrict the motion of ith mass.

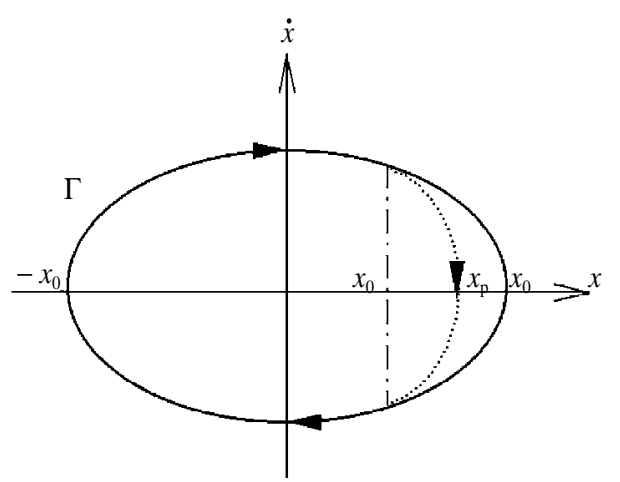

$-x_{0} \sim x_{c}$ : Displacement interval for breathing crack with rigid boundary

$-x_{0} \sim x_{p}$ : Displacement interval for breathing crack with elastic boundary

$-x_{0} \sim x_{0}$ : Displacement interval for opening crack mode in free vibration

Fig. 2. A bilinear motion plotted on the phase plane as a comparison of motion loci for opening crack mode and breathing crack mode. tory in the phase plane referring to Figure 2. $T_{-i}$ is the period of free motion from $-x_{0}$ to $x_{c i}$ and $T_{+i}$ is the period of constrained motion from $x_{c i}$ to $x_{p}$.

\section{(1) $T_{-i}$ period of free motion}

Since $d t=\frac{d x_{i}}{d x_{i}} d t=\frac{d t}{d x_{i}} d x_{i}=\left(\frac{1}{x_{i}}\right) d x_{i}$ and

$$
\begin{aligned}
\frac{1}{x_{i}} & =\left(\kappa_{i j} \sqrt{\left(x_{0 j}^{2}-x_{j}^{2}\right)}\right)^{-1}=\left(\kappa_{i j}^{-1} \frac{1}{\sqrt{\left(x_{0 j}^{2}-x_{j}^{2}\right)}}\right) \\
& =\left(\varsigma_{i j}^{-1} \frac{1}{\sqrt{\left(x_{0 j}^{2}-x_{j}^{2}\right)}}\right)
\end{aligned}
$$

The half period for free motion $T_{-i}$ can be obtained from the integration in the time domain.

$$
\begin{aligned}
T_{-i} & =2 \varsigma_{i j} \int_{x_{c i}}^{-x_{0 i}} \frac{d x_{i}}{\sqrt{\left(x_{0 j}^{2}-x_{j}^{2}\right)}} \\
& =2 \varsigma_{i j} \int_{x_{c j}}^{-x_{0 j}} \frac{1}{\sqrt{\left(x_{0 j}^{2}-x_{j}^{2}\right)}} \frac{\partial x_{i}}{d x_{j}} d x_{j}
\end{aligned}
$$

where $[\varsigma]=\left([m]^{-1}[k]\right)^{\frac{1}{2}}$

By letting $\frac{\partial x_{i}}{\partial x_{j}}=\frac{d x_{i}}{d t} \frac{d t}{d x_{i}}=\frac{\dot{x}_{i}}{x_{j}}=\frac{\frac{d}{d t}\left(x_{0 j} \sin \omega t\right)}{\frac{d}{d t}\left(x_{0 j} \sin \omega t\right)}=\frac{x_{0 j}}{x_{0 j}}$

, Eq. (10) becomes

$$
T_{-i}=2 \varsigma_{i j} \int_{x_{c j}}^{-x} \frac{1}{\sqrt{\left(x_{0 j}^{2}-x_{j}^{2}\right)}} \frac{x_{0 i}}{x_{0 j}} d x_{j}
$$

After integrating Eq. (11), we get

$$
T_{-i}=2 \varsigma_{i j} \frac{x_{0 i}}{x_{0 j}}\left[\frac{\pi}{2}-\sin ^{-1}\left(-\frac{x_{c j}}{x_{0 j}}\right)\right]
$$

Finally, the time period of free motion can be represented in matrix form as

$$
\begin{aligned}
& T_{-i}=2 x_{0 i}\left\langle\begin{array}{lllll}
\varsigma_{i 1} & \varsigma_{i 2} & \cdots & \cdots & \varsigma_{i n}
\end{array}\right. \\
& \left\{\begin{array}{c}
\frac{1}{x_{01}}\left[\frac{\pi}{2}-\sin ^{-1} \frac{x_{c 1}}{x_{01}}\right] \\
\frac{1}{x_{02}}\left[\frac{\pi}{2}-\sin ^{-1} \frac{x_{c 2}}{x_{02}}\right] \\
\vdots \\
\vdots \\
\frac{1}{x_{0 n}}\left[\frac{\pi}{2}-\sin ^{-1} \frac{x_{c n}}{x_{0 n}}\right]
\end{array}\right\}
\end{aligned}
$$




\section{(2) $T_{+i}$ period of constrained motion}

In the constrained motion, if the mass oscillates with enough amplitude, it will contact with the elastic boundary by a depth. Generally, the depth of penetration depends on the contact stiffness of the interface between the two contact bodies, which can be described by the following term $\delta$ according to the studies by Butcher[3] and Todoo [21], respectively,

$$
\begin{aligned}
& \delta=\left(x_{0} / \alpha\right) \sqrt{1-\left(x_{0 i}^{2} / x_{0 j}^{2}\right)\left(1-1 / \alpha^{2}\right)}, \\
& \alpha=\sqrt{1+\left(x_{0 i} / x_{0 j}\right)}
\end{aligned}
$$

The period of constrained motion from $x_{c i}$ to $x_{p}$ can therefore be obtained by intergrating the term $T_{+i}$ in Eq. (10) with $x_{p}=x_{c i}+\delta$, that is

$$
T_{+i}=2 \varsigma_{i j} \frac{x_{0 i}}{x_{0 j}} \frac{1}{\alpha}\left[\frac{\pi}{2}-\sin ^{-1}\left(-\frac{x_{c j}}{x_{0 j}} \sqrt{\left.1-\frac{x_{c j}^{2}}{x_{0 j}^{2}}\left(1-\frac{1}{\alpha^{2}}\right)\right)}\right]\right.
$$

\section{(3) $T_{i}$ total period of bilinear motion}

The total period of the bilinear motion can be found by adding the partial periods of each motion of the two regions, free motion period (12) and constrained motion period (15).

$$
\begin{aligned}
& T_{+i}=2 \varsigma_{i j} \frac{x_{0 i}}{x_{0 j}}\left[\frac{\pi}{2}-\sin ^{-1}\left(-\frac{x_{c j}}{x_{0 j}}\right)\right. \\
& +\frac{1}{\alpha}\left(\frac{\pi}{2}-\sin ^{-1}\left(-\frac{x_{c j}}{x_{0 j}} \sqrt{1-\frac{x_{c j}^{2}}{x_{0 j}^{2}}\left(1-\frac{1}{\alpha^{2}}\right)}\right)\right]
\end{aligned}
$$

The bilinear natural frequency is obtained as

$$
\Omega_{i}=\frac{2 \pi}{T_{i}}
$$

\section{ANALYSIS OF A CRACKED STRUCTURE}

In this study, two cases were investigated to understand the significance of the current method. Section 1 discusses a beam with a single-edge crack analyzed by two different methods. Section 2 discusses a bladeshaped structure with a single-edge crack. Due to the presence of the crack, there is a $\sqrt{1 / r}$ singularity in the stress field at the crack tip [5]. Usually, quarter point (singular) elements are used to model the singular behavior in the stress field. However, in this particular analysis, global changes in the dynamic behaviors of the beam, rather than local variations in the stress field due to the presence of the crack, are expected; the regular element was proven to predict similar results as that obtained from the model meshed with a singular element near the crack site [5]. Therefore, regular elements are adopted in calculations from this point on.

\section{Analysis of a cracked beam structure}

To demonstrate the validity of the current method, two cracked beam models from our previously published works are used to investigate the effect of cracks on dynamic behavior.

\section{(1) Comparison with the bilinear method}

A cracked aluminum prismatic cantilever beam is generated [5]. The length of the beam is $235 \mathrm{~mm}(\mathrm{~L})$, width $7 \mathrm{~mm}(\mathrm{~W})$, and height $23 \mathrm{~mm}(\mathrm{H})$. Young's modulus is $72 \mathrm{GPa}(\mathrm{G})$, density $2,800 \mathrm{Kg} / \mathrm{m}^{3}(\rho)$ and Poisson's ratio $0.3(v)$. A finite element model of the cracked beam with a single-edge crack at mid-span is shown in Figure 3. The computed results of the normalized natural frequencies versus the normalized crack depth of the cracked beam are shown in Figure 4. Comparative results obtained by Chondros et al. [5] by experiment are also depicted. In Figure 4, it can be found that a crack with serious depth results in a signifi-

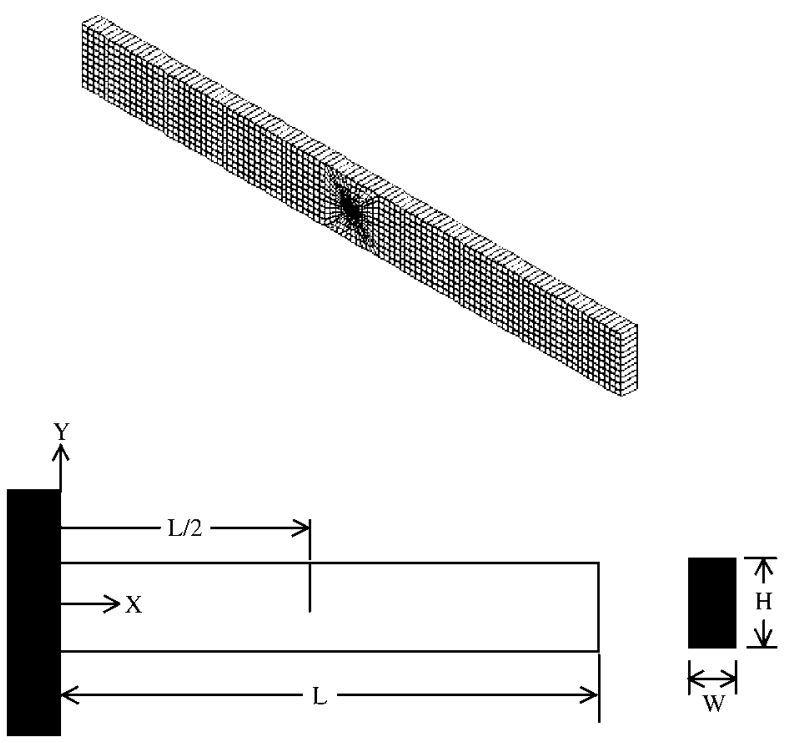

Fig. 3. The finite element model of a cracked beam with refined mesh at crack site. 
cant decrease in vibrational frequency owing to the decrease of beam stiffness at the crack site. The results of the current method also reveal that the natural frequency for a beam with a breathing crack is higher than that with an open crack. It is worth to note that this cracked beam has a first bending vibrational mode similar to that brought about in an experiment performed by Ghondros [5].

\section{(2) Comparison with the torsional spring method}

The presence of the crack causing a local flexibility to the beam can be expressed by a linear torsional constant $k_{t}$. The evolution of the constant $k_{t}$ is a matter of variable complexity depending on the structure. Rice \& Levy [19] showed that a torsional spring constant due to the crack can be obtained as: $k_{t}=\frac{E w^{3}}{72\left(1-v^{2}\right) F(a / w)}$ where the spring constant for a Plexiglas beam is a function of the crack depth ratio $a / w$. For further comparison here, the beam has the same geometry and material properties as those used by Bamnions and Trochides [1]. A comparison of the results of current FEM simulations and those predicted using the torsional spring method by Bamnions and Trochides [1] is presented in Figure 5. From this comparison, it can be seen that there is great consistency in the predictions of the dynamic behavior of the cracked beam. However, slight differences can be found between these results. This can be ascribed to the fact that the contact stiffness

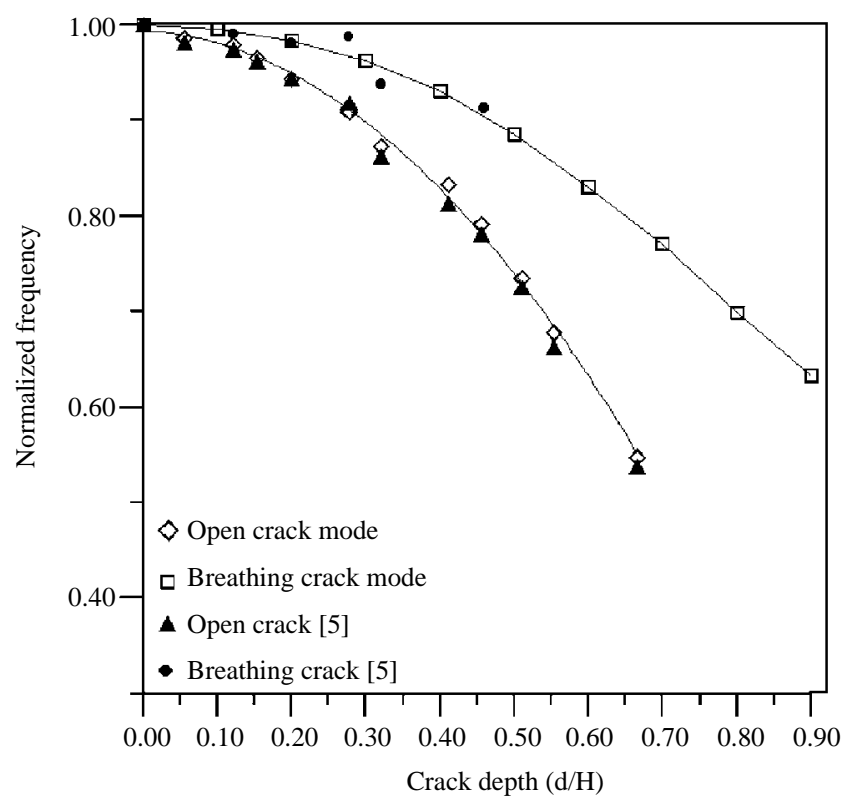

Fig. 4. Variation of the first normalized vibration frequency as a function of crack depth, compared with the results obtained in reference [5]. owing to the mutual interference of crack interface in the crack closing period was not considered in the torsional spring method [8], but dealt with a compatibility condition of displacement assumed at the interface. This may lead to an underestimation of beam stiffness across the crack site and hence reduce the vibrational frequency. However, for a breathing crack, the crack surface is allowed to move freely during the opening period; while in the closing period the displacement is restricted by the crack sides coming into contact. This obviously shortens the motion path cycle in this period. Since the period the crack is closed contributes a part of the time needed to complete a motion cycle, it follows that this will increase the vibrational frequency of this half cycle with its constrained motion. Such an effect of constrained motion on vibration has been considered in this study by estimating the time period in the time domain.

\section{Analysis of a blade-shaped component crack}

A blade-shaped component crack may cause local change in the flexibility $[2,7]$. The FEM model shown in Figure 6 was divided into 10 different location points along the axial direction and divided into 7 location points along the lateral direction $b$. The material properties are as follows: $E=207 \mathrm{GPa}, \rho=\mathrm{Kg} / \mathrm{m}^{3}, \gamma=0.3$. The normalized natural frequency corresponding to different crack depths and positions can be predicted by current methods and their relationships can be further depicted in three-dimensional diagrams as shown in Figure 7. From these figures, it is found that the variations of natural frequency can be expressed as a function of the crack depth for some crack positions. As expected, the frequency variation decreases with the

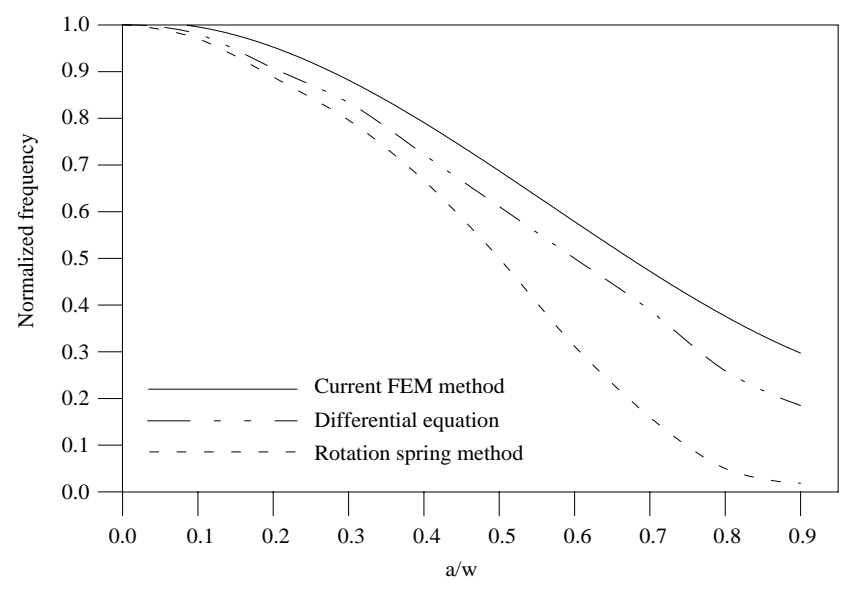

Fig. 5. Variation of the first normalized vibration frequency as a function of crack depth, compared with the results obtained in reference [1]. 
depth of the crack. When the crack grows along a lateral direction, it implies that for a given crack location the reduction in natural frequencies increases with the increment of the crack depth. For a crack located at midpoint $(a=5)$ the natural frequency decreases about $50 \%$ for the first mode and $13 \%$ for the second mode. Therefore, the effect of crack depth on the dynamic characteristics changes with the crack axial position initiated in the blade-shaped component structure. Since the major stiffness is dominated by the clamped part of the cantilever blade-shaped component structure, as a consequence, a crack occurring near the fixed end of the blade will result in a substantial decrease in the frequency compared to that near the free end. For a crack with a constant depth $(b=5)$ the natural frequency decreases about $7 \%$ for the first mode and $12 \%$ for the second mode. For a crack embedded in a blade-shaped component structure, it can be found from Figure 8 that the natural frequency assessed in the breathing crack mode is always higher than the crack assessed in the opening state. This difference can be ascribed to the fact that the contact stiffness changes owing to the mutual interference of crack interfaces. That was not considered in the crack opening mode. A breathing crack may increase frequencies due to decreases in the period of constrained motion. As described previously the current method provides more precise and correct crack information than others when detecting crack depth and location. Results derived from the current method also indicate that both the crack location and depth indeed influence the dynamic behavior of the
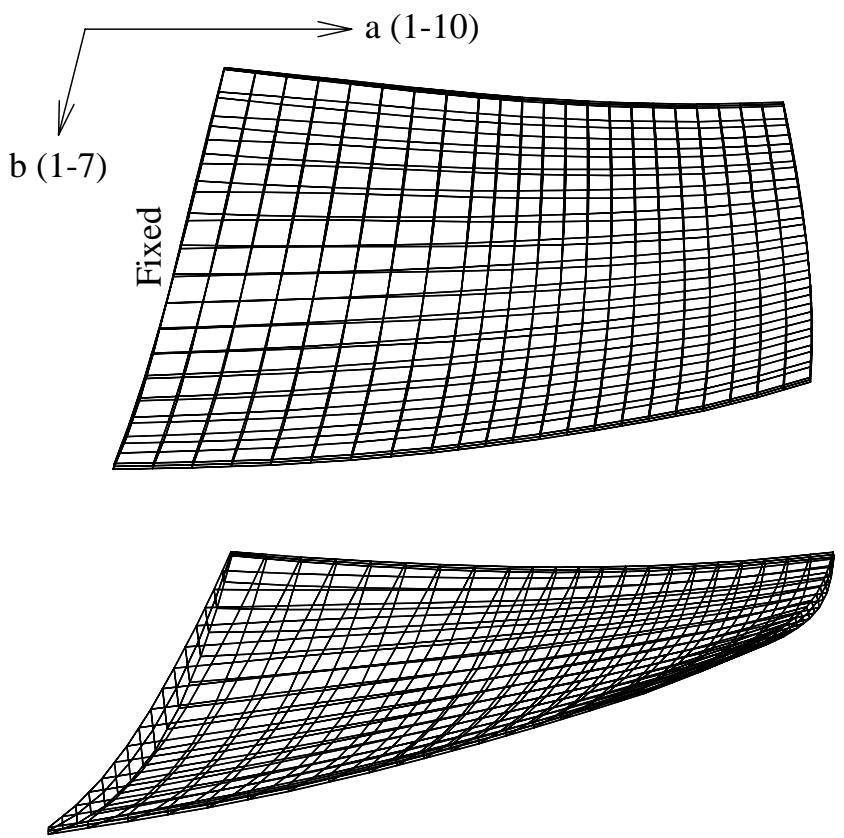

Fig. 6. The finite element model of a rotor blade. crack structure to different degrees. The existence of a crack can thus be confirmed by measuring the change in natural frequencies and comparing them to that measured for an intact structure.

\section{CONCLUSION}

This study focuses on the dynamic behaviors of cracked structures using the finite element approach (FEA). In order to get rid of the complexity of nonlinearity of cracked structures, many researchers have assumed that the crack surface is persistently in an open state or the opposing sides of the crack do not come into contact during vibration. Actually, such a hypothesis contradicts the reality of the situation. Therefore, the vibration model of breathing cracks is employed to understand real vibrational behavior. The vibrational cycle of a breathing crack is non-symmetric including its open and closed period. In the closed period of the crack, the motion will be restricted to a narrow region constrained by mutual interference of the crack sides. In order to obtain the overall period for the purpose of

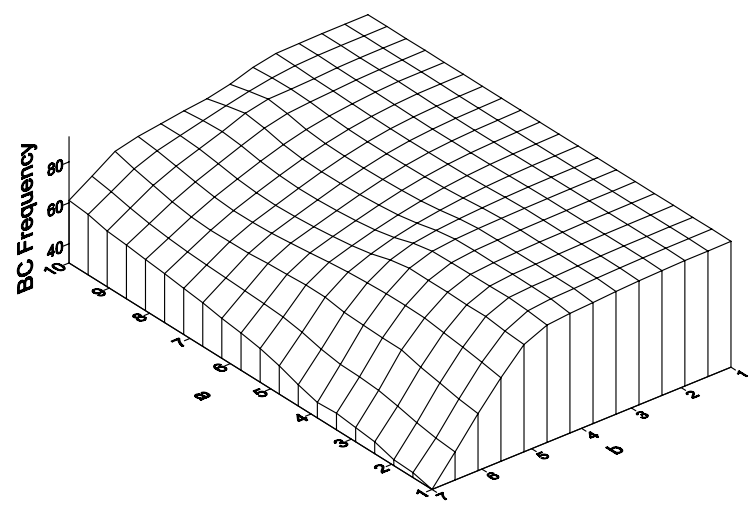

(a) First mode

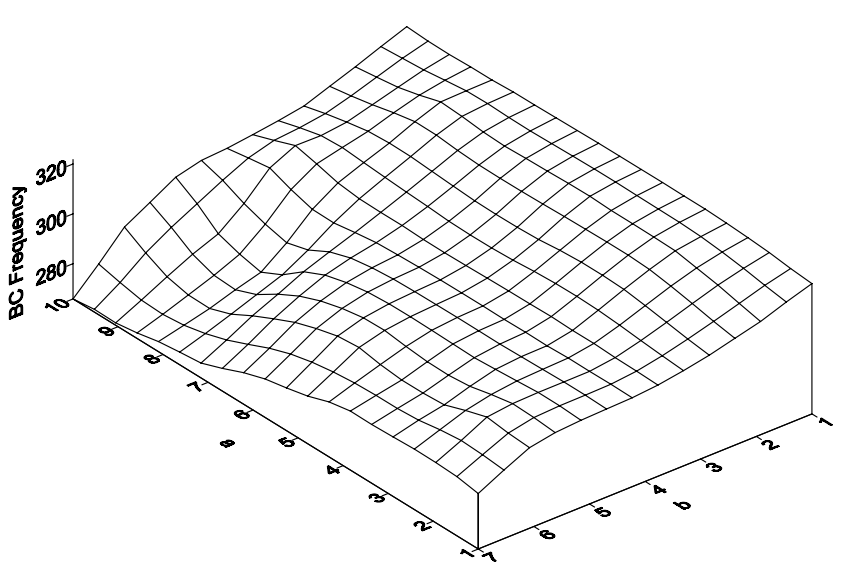

(b) Second mode

Fig. 7. Frequencies versus crack depth and crack position for a cracked blade (a) first mode; (b) second mode. 


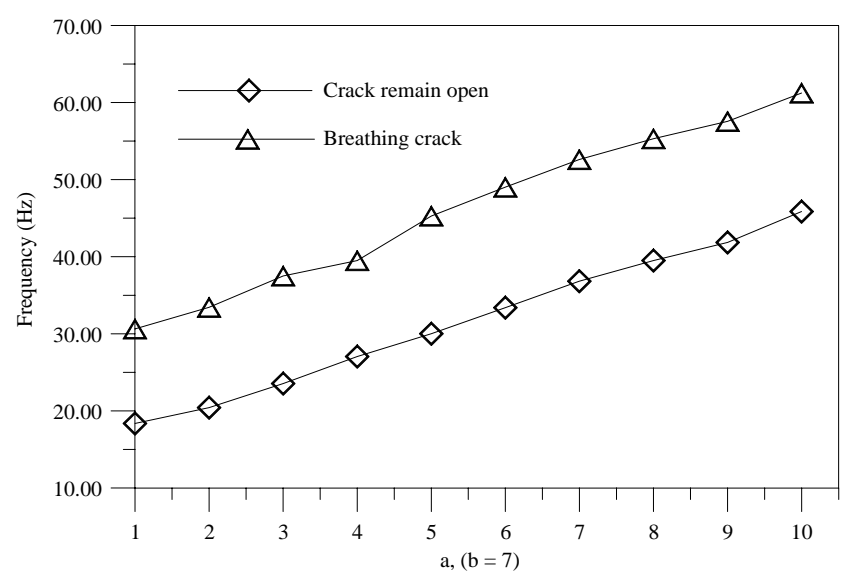

(a)

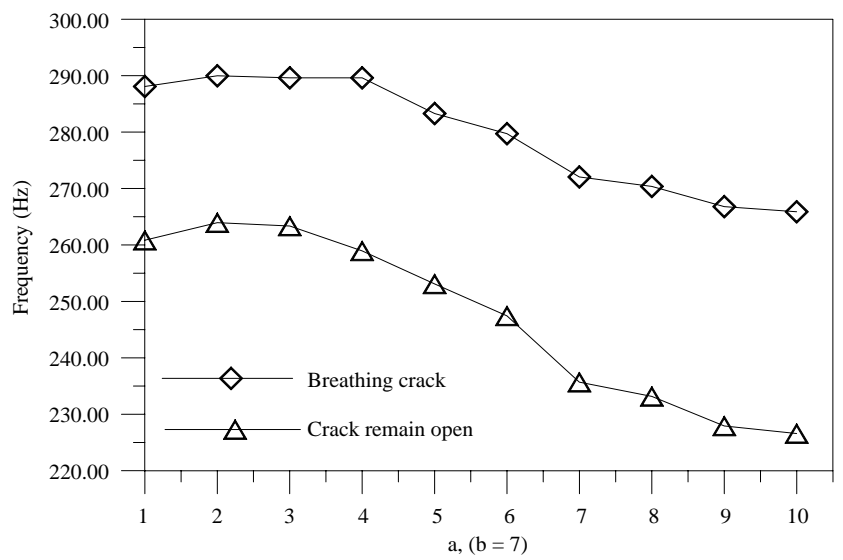

(b)

Fig. 8. Comparison natural frequencies of breathing crack and crack remain open (a) first mode; (b) second mode.

finishing the unsymmetrical motion cycle, this study considers the contact effect as being a bilinear theory on crack sites in computations. All FEA formulae derived in the time domain are applied in order to estimate the above two periods. To demonstrate the validation of the current method, two cracked beam models coming from previously published works are used to investigate the effect of cracks on dynamic behavior. Current results obviously reveal that both the crack location and depth indeed influence the dynamic behavior of the cracked beam to different degrees. In addition to this, comparison of the current simulation results and those available in the literature has shown a significant consistency when evaluating the variations in natural frequency. Slight differences can be ascribed to the fact that the contact effect in the closing period of the crack has not been considered in some of the other literature. Results derived from the current method also indicate that both the crack location and depth indeed influence the dynamic behavior of the crack structure to different degrees.
Thus the existence of a crack can be confirmed by measuring the change in natural frequencies. Different depths of cracks and positions in the beams structure will change the natural frequency from $5 \%$ to $60 \%$. These apparent and identifiable variable quantities can be a basis for reference for on-line monitors of cracks or the safe design of structures. It is believed that the current method can provide a reliable and accurate technique for the detection of cracks occurring in other damaged structures such as blade-shape components.

\section{REFERENCES}

1. Bamnios, G. and Trochides, A., "Dynamic Behaviors of a Cracked Cantilever Beam," J. Appl. Acoust., Vol. 45, pp. 97-112 (1995).

2. Bernstein, H.L. and Allen, J.N., "Analysis of Cracked Gas Turbine Blades.," J. Eng. Gas Turb. Power, Vol. 114, pp. 293-301 (1992).

3. Butcher, E.A., "Clearance Effects on Bilinear Normal Mode Frequencies," J. Sound Vib., Vol. 224, No. 2, pp. 305-328 (1999).

4. Carson, R.L., "An Experimental Study of the Parametric Excitation of a Tensioned Sheet with a Crack Like Opening," Exp. Mech., Vol. 14, No. 2, pp. 452-458 (1974).

5. Chondros, T.G. and Dimarogonas, A.D., Yao., "Vibration of a Veam with a Breathing Crack," J. Sound Vib., Vol. 239, No. 1, pp. 57-67 (2001).

6. Fernandez, S.J. and Navarro, C., "Fundamental Frequency of Cracked Beams in Bending Vibrations An Analytical Approach," J. Sound Vib., Vol. 256, No. 1, pp. 17-31 (2002).

7. Galvele, J.R., "Surface Mobility Stress Corrsion Cracking Mechanism of Steels for Steam Turbine Rotor.," $J$. Corros. Sci., Vol. 30, pp. 955-958 (1990).

8. Gounaris, G. and Dimarogonas., "A Finite Element of a Cracked Prismatic Beam for Structural Analysis," Comput. Struct., Vol. 28, No. 3, pp. 309-313 (1988).

9. Gudmundson, P., "The Dynamic Behavior of Slender Structures with Cross-sectional Cracks," J. Mech. Phys. Solids, Vol. 31, No. 1, pp. 329-345 (1983).

10. Hjelmstad, K.D. and Shin, S., "Crack Identifcation in a Cantilever Beam from Modal Response," J. Sound Vib., Vol. 198, No. 1, pp. 527-545 (1996).

11. Khiem, N.T. and Lien, T.V., "A Simplified Method for Natural Frequency Analysis of a Multiple Cracked Beam," J. Sound Vib., Vol. 245, No. 4, pp. 737-751 (2001).

12. Khiem, N.T. and Lien, T.V., "The Dynamic Stiffness Matrix Method in Forced Vibration Analysis of Multiple-cracked Beam," J. Sound Vib., Vol. 254, No. 3, pp. 541-555 (2002).

13. Kisa, M., "Free Vibration Analysis of a Cantilever Com- 
posite Beam with Multiple Cracks," J. Compos. Sci. Tech., Vol. 64, No. 9, pp. 1391-1402 (2004).

14. Krawczuk, M. and Ostachowicz, W.M., "Transverse Natural Vibrations of a Cracked Beam Loaded with a Constant Axial Force," J. Vib. Acoust., Vol. 115, pp. 524-528 (1993).

15. Lee, H.P. and Ng, T.Y., "Natural Frequencies and Modes for the Flexural Vibration of a Cracked Beam," $J$ Appl. Acoust., Vol. 42, No. 2, pp. 151-163 (1994).

16. Lin, H.P., "Direct and Inverse Methods on Free Vibration Analysis of Simply Supported Beams with a Crack," Eng. Struct., Vol. 26, No. 4, pp. 427-436 (2004).

17. Murphy, K.D. and Zhang, Y., "Vibration and Stability of a Cracked Translating Beam," J. Sound Vib., Vol. 237, No. 2, pp. 319-335 (2000).
18. Nandi, A. and Neogy, S., "Modelling of a Beam with a Breathing Edge Crack and Some Observations for Crack Detection," J. Vib. Control, Vol. 8, No. 5, pp. 673693 (2002).

19. Rice, J.R. and Levy, N., "The Dynamic Behavior of Slender Structures with Cross-sectional Cracks," J. mech. Phys. Solids, Vol. 31, pp. 329-345 (1983).

20. Rizos, P.F., Aspragathos, N., and Dimarogonas, A.D., "Dentification of Crack Location and Magnitude in a Cantilever Beam from the Vibration Mode," J. Sound Vib., Vol. 138, pp. 381-388 (1990).

21. Todd, M.D. and Virgin, L.N., "Natural Frequency Computations of Impact Oscillator," J. Sound Vib., Vol. 194, No. 3, pp. 452-460 (1996). 